\title{
XIV. Molecular electronics
}

Microelectronics and nanoelectronics are made by top-down approaches. Another way to manufacture nanoscale devices is to use atoms, single or group of molecules, carbon nanotubes, metallic or semiconductor nanowires as the elementary components and find methods to assemble them in the right way to perform electronic functions. This is the essence of molecular electronics called also moletronics. Sometimes, the term molecular electronics is restricted to the use of single molecules only but, for applications, it is better to use an extended definition involving nanoscale components. It is not an easy task to develop bottom-up approaches because nanoscale objects already contain a large number of molecules and atoms and the main issue is to assemble them correctly and quickly in the right way.

Organic molecules are often used in molecular electronics because they offer an enormous number of possibilities. One advantage of bottom-up approaches is the ability to choose starting material from large number of organic molecules that are already characterized. Another is that their properties can be tailored using chemical synthesis techniques. Since specific molecules can be precisely produced by chemical synthesis, the bottom-up process can be initiated with identical molecules. However, assembling them to get a nanodevice is not straightforward. Self-assembly is often used because it provides the ability to manufacture complex systems at a high speed.

The fundamental question of molecular electronics science is to understand how electrons move through a molecule. Historically, laboratory studies have long addressed that question. However, the field of molecular electronics really started in 1974, when A.Aviram and B.Ratner first proposed the idea of a molecular rectifier.

\section{Electronic conduction}

From the fundamental point of view, the first aspect to be understood is how a charge moves from one side of a molecule to the other. Two things are needed for electron conduction in inorganic materials. First, it is necessary to have a large number of orbitals interacting strongly with each other so that a band structure can be obtained (see figure 111). This is obtained in lattice structures. Second, there should be enough electrons to fill these bands. These two conditions are fulfilled by metals and semiconductors. 


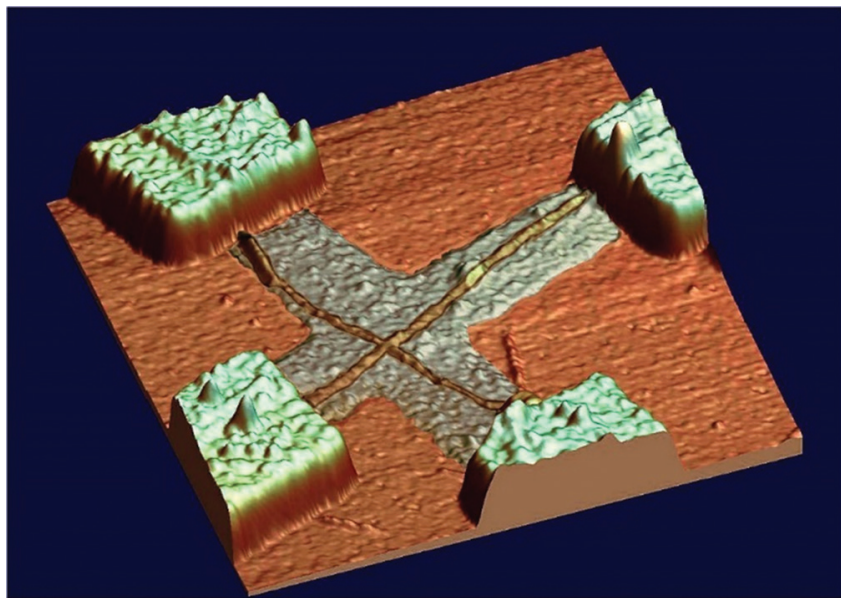

Figure 115. Crossover of two carbon nanotubes deposited on silica. The aim of this experiment, a joint work by CEA and MOTOROLA, was to illustrate the production of nanotube circuits by selfassembly. The gray strips are molecular glue used to position the nanotubes. Image courtesy of CEA/LETI (France), Clef CEA n52.

In the case of organic molecules, delocalized electronic states are necessary. In addition, other conditions may be required. For example, conjugated polymers are conducting only if they are doped.

A second aspect is to study and understand how a current passes through a molecule that is connected at each end to an electrode. An important issue is how to electrically connect a molecule to the external world. It is necessary to do that to measure electrical properties and to build complex electronic devices. Gold or platinum are normally used to manufacture electrodes because they are resistant to oxidation and degradation. Sulfur or amine molecules can be used to attach the molecules to gold. Molecules with unbonded pairs of electrons are often used with both gold and platinum electrodes. Figure 115 shows an example of a laboratory experiment to investigate the crossover of two nanotubes.

Different mechanisms are involved in the electric conduction of molecules. Conductivity can arise from quantum tunneling or by hopping conduction in which a charge carrier has the ability to move between localized states.

\section{Difficulties}

Even if performing a logical function is possible with a molecule, further conditions are necessary for this function to be realized or included 
in a more complex electronic system. Consider, for example, one of the simplest functions that can be imagined: a molecular switch. This could be a molecule that can exist in two different conformational states. One state would correspond to the switch "on", the other to the switch "off". In order to be useful, the molecular switch should fulfill the following conditions:

- The switch should change state on demand and not spontaneously.

- The transition from "on" to "off" has to be reversible otherwise that would be a one shot switch

- The switch has to be addressable, which means that, if there are a large number of switches available, we have to be sure to activate this switch and not another one. Since the switch molecule is generally positioned among other molecules, one has to be sure that it stays in place and does not move to another place due to thermal motion.

- The switch should be inert with respect to chemical reaction with other molecules and remain stable under the ambient external conditions.

All these conditions put heavy constraints on the molecular properties and on the architecture of the device that makes it difficult for this field to reach large scale development.

\section{Molecular wires}

Electronic conduction in molecules is possible if there are overlapping orbitals allowing a delocalization of electrons over the whole molecule. Electrons moving in delocalized electron orbitals do not belong to a single bond or atom, but rather to a group of atoms. Such molecules can be used as molecular wires. The simplest structure that can be designed consists of an organic molecule that is a chain of alternating single (two electrons, sigma) and double (four electrons, pi) bonds (Figure 116, top). Such a molecule is said to be a conjugated system. In this case, the $\pi$ electrons are delocalized over the whole chain. More complicated structures involving aromatic components, i.e., rings of conjugated bonds, can also be made.

In Figure 117 the six carbon atom structures $\mathrm{C}_{6} \mathrm{H}_{6}$, benzene and $\mathrm{C}_{6} \mathrm{H}_{12}$, cyclohexane are depicted. The benzene molecule is a conjugated aromatic molecule. Although a benzene ring cannot be used alone in molecular electronics, one or several benzene rings can be associated to other functional groups to make complex molecules that can be used for 


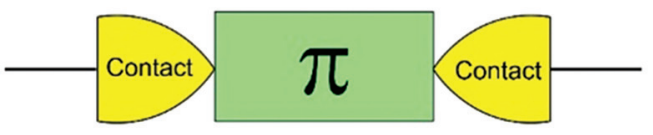

Molecular wire

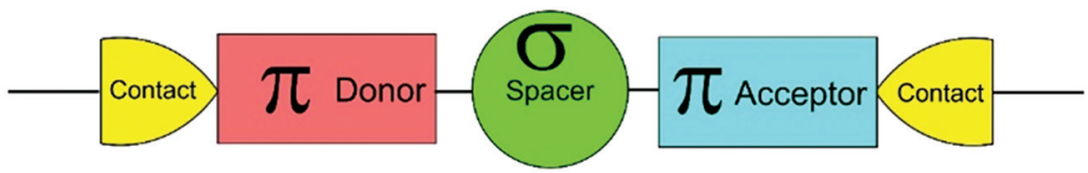

\section{Molecular diode}

Figure 116. Top of the figure: principle of a molecular wire. The rectangle named $\pi$ corresponds to the molecular wire. It is a molecule with delocalized $\pi$-electrons. Bottom of the figure: Basic principle of a molecular diode. The circle named $\sigma$ is a part of the molecule with only $\sigma$ electrons (electrons localized in bonds).

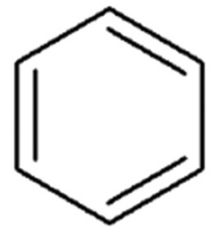

Benzene

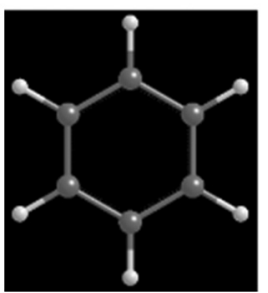

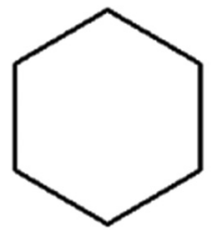

Cyclohexane

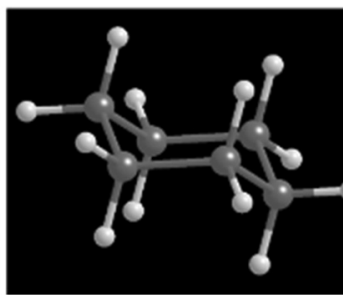

Figure 117. Benzene $\left(\mathrm{C}_{6} \mathrm{H}_{6}\right)$ and cyclohexane $\left(\mathrm{C}_{6} \mathrm{H}_{12}\right)$ molecules. Benzene is a conjugated molecule in which $\pi$ electrons are delocalized over the whole ring (above and below). The benzene molecule is planar. Cyclohexane has only $\sigma$ bonds and is non-planar for this reason.

electronic conduction. It has also been observed that the conductivity of some organic molecules can change with the intensity of an applied electric field.

In the field of inorganic semiconductors, the valence and conduction bands play a preeminent role. The energy difference between the bottom 
of the conduction band and the top of the valence band is the energy gap of the semiconductor. In the field of organic conductors, the important bands are the HOMO (highest occupied molecular band) and the LUMO (lowest unoccupied molecular band). Loosely speaking, the HOMO is similar to the valence band maximum of the inorganic semiconductors and the LUMO is similar to the conduction band minimum.

Carbon nanotubes are macromolecules with high mechanical strength and lengths which are much larger than their transverse dimensions. They have conductivities ranging from metallic to semiconducting depending upon their structure (see chapter 7). Their properties make them a good choice for connections to external electrodes.

Wiring in molecular resistors is easier than molecular wiring to external electrodes. An isolating molecule can be put between two conducting molecules, for instance. Electrically isolating molecules are more common than conducting molecules. Alkanes, which are made of $\sigma$-bonds only are good examples of isolating molecules.

\section{Molecular diodes and transistors}

A molecular diode is a two-terminal device used as a rectifier in electronics. It conducts electricity in one direction only. It is possible to design a molecular diode as indicated in the bottom part of figure 116. It can be a molecule with the donor part separated from an acceptor part by a non-conducting part (spacer) playing the role of a junction barrier. The donor and acceptor parts have delocalized $\pi$-electrons while the spacer has $\sigma$-electrons only. Electrons can go through the spacer by tunneling.

Transistors are 3-terminal devices. They have the ability of amplifying and switching electronic signals. They are needed in molecular electronics to develop the same functionalities as those developed in micro and nanoelectronics. There are basically two ways to develop 3-teminal devices. The first one is to synthesize a molecule with three different ports that can be each connected to three different electrodes.

The second one is to attach a third terminal close to, but not in contact, with a 2-terminal molecule. This third terminal plays the role of a gate and applying a potential to it changes the electrostatic state of the 2-terminal molecule allowing transition from a Coulomb blockade regime to a conducting regime. Such a device has to be operated at very low temperature to avoid any thermal excitation. 


\section{Conductive polymers}

Polymers have been a revolution in the field of materials. They are usually cheap and easy to manufacture. Conventional polymers are good insulators for heat and electricity. Conjugated polymers are a class of polymers that can become conductive when partially oxidized or reduced by doping. They are often named intrinsically conducting polymers. Their conductivity can be that of a metal or that of a semiconductor depending on the nature of the polymer, and its subsequent treatment. For example, the conductivity of polyacetylene can be increased by a factor of $10^{8}$ by oxidative doping with iodine vapor. Doping by chlorine or bromine is also possible. Polyacetylene is a very interesting intrinsically conducting polymer for fundamental studies but is unstable in ambient conditions. It was the first known example of a synthesized conducting polymer. A.J.Heeger, A.MacDiarmid and H.Shirakawa were awarded the Nobel Prize in Chemistry, in 2000, for their discovery of conducting polymers, published in 1977.

The addition of a donor or an acceptor molecule to the polymer is called doping in analogy to techniques used with inorganic semiconductors. Actually, in the case of conjugated polymers, doping induces an oxidation-reduction reaction. In a first step, there is the formation of a cation or anion radical called a soliton or polaron. In a second step, there is either a second electron transfer with the formation of a di-cation or di-anion called a bipolaron, or charged transfer complexes can be formed. Dopants can be electron acceptors such as $\mathrm{Br}_{2}, \mathrm{I}_{2}, \mathrm{H}_{2} \mathrm{SO}_{4}$, $\mathrm{AsF}_{5}$, etc., or electron donors such as alkali metals. Compared to inorganic semiconductors where the concentration of dopants is small (smaller than $1 \%$ ), the concentration of dopants in conductive polymers is large, typically in the range of $20-40 \%$.

The electronic properties of conductive polymers depend to a large part on the value of their bandgap. It is therefore important to be able to adjust this quantity on demand. Several parameters influence the bandgap:

- Increasing the degree of polymerization decreases the value of the bandgap

- The bigger the alternating structure of single and double bonds, the larger is the bandgap

- The presence of aromatic parts in the structures increases the bandgap and stabilizes the polymer.

- It is also possible to modulate the bandgap by playing with the conformation of the monomer molecules or by introducing substituent groups. 


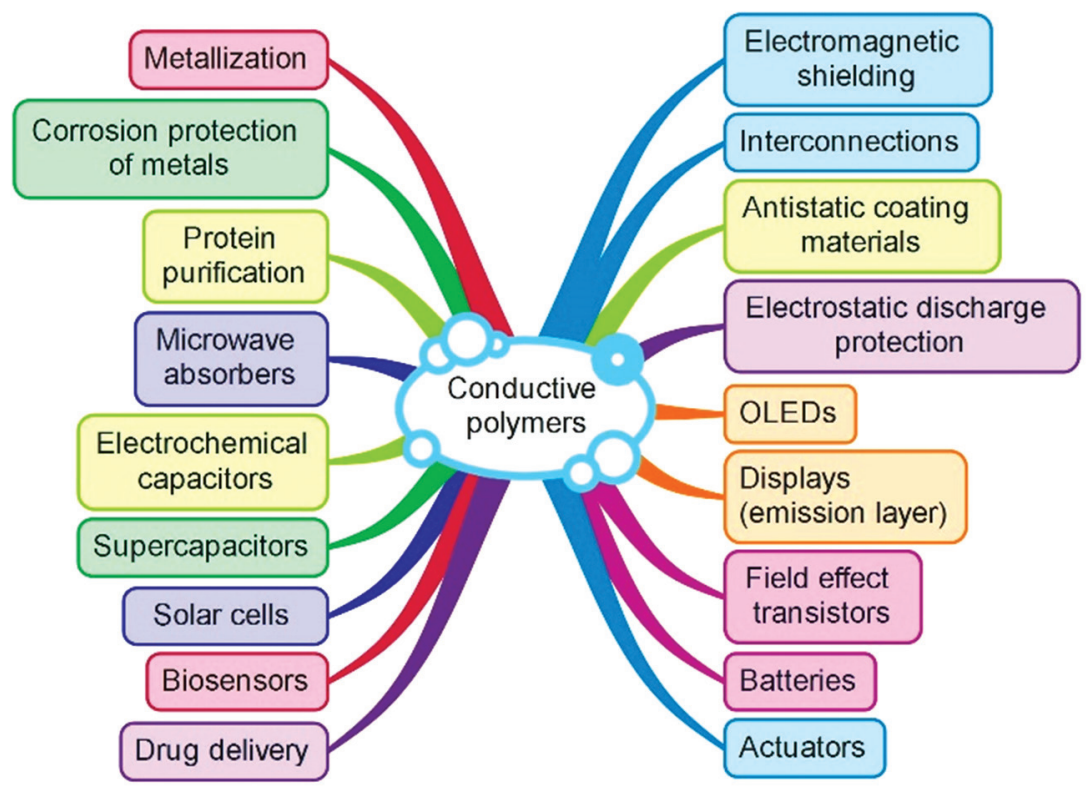

Figure 118. Some applications of conductive polymers.

Several conductive polymers are known: polyacetylene, polyaniline, polypyrrole, polythiophene, etc. Compared to usual polymers, conductive polymers do not possess the same mechanical properties and are not thermoformable. Conductive polymers have several applications that already exist but also a large number of potential applications. Both are shown in figure 118.

As an illustration of some applications:

- It is possible to develop polymer-based photovoltaic devices in which incident photons generate excitons or bound electron-hole pairs in a small molecule layer and in a polymer layer. These excitons dissociate at the small molecule-polymer interface to create free electrons and holes that will generate a current in the device.

- It is possible to manufacture touch sensors, electrochromic pixel displays, biochemical sensors, LEDs or electrical supercapacitors based on conductive polymers.

- Polypyrrole can be used to create electrochemical capacitors. Conductive polymers can also be used in microwave absorbers for radar discretion.

Intermediate between single molecules (monomers) and polymers, oligomers are also often used in molecular electronics. While polymers 


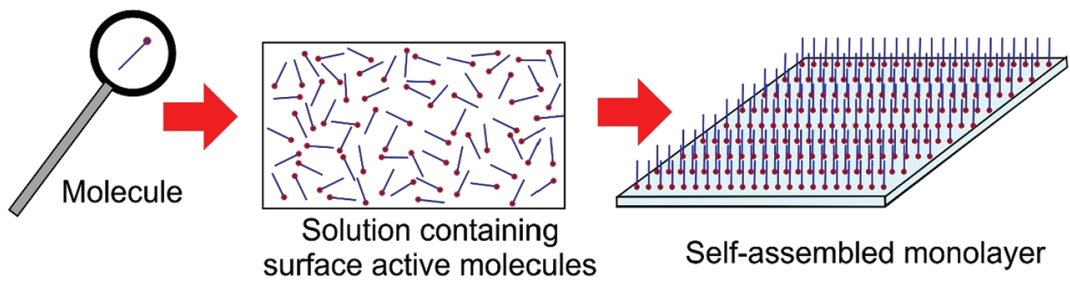

Figure 119. Illustration of self-assembled monolayer deposition via surface active molecules.

consist of a large number of monomer units, oligomers are made of a few monomer units only. They are interesting because they can be synthesized step by step with a complete control of the structure and of their architecture.

\section{Self-assembled monolayers}

Monolayers are important structures useful in many applications. We have already presented the Langmuir-Blodgett method to build monolayers in chapter 6 . There are other techniques to make self-assembled monolayers (called often SAMs). SAMs can be formed by adsorption of a solution or vapor of surface active molecules on a solid surface (figure 119). The molecules spontaneously orientate towards the substrate surface and arrange in an ordered layer corresponding to thermodynamic equilibrium. It turns out that the functional end groups of the initial molecules chemically react with the substrate.

Compared to Langmuir-Blodgett films, where the monolayers correspond to physisorbed bonds, SAMs are more stable because they are based on a strong chemisorption of the head group of the molecules. Self-assembled monolayers open the possibility of making dense functional systems with possible potential applications as switches or memories.

\section{Summary}

Molecular electronics and nanoelectronics are complementary. The former rely on a bottom-up approach while the latter employs a top-down approach. Since nanoscale devices still contain a very large number of single molecules or atoms, it is necessary to use self-organized methods to build objects from single elements at a decent rate. 
Except for special cases such as conductive polymers, for example, molecular electronics is still in its infancy. Even though elementary devices have been made in the laboratory, we are still far from being able to replace the complex micro or nanoelectronic systems that modern society relies upon.

In molecular electronics is that it is possible to have complete control on the molecules forming the building blocks. Furthermore, it is also possible to graft specific functions on a given basic molecule to tailor specific properties of the molecules. These modified molecules are useful as building blocks for the construction of nanoscale devices. Another interest in developing molecular electronics is to better understand the fundamental mechanisms involved in electronic transport processes.

The near future for molecular electronics, and also for nanoelectronics, is that some of the functions developed will become part of larger microdevices. Complex systems will in fact become hybrid systems containing micro, nano and molecular components. 